\title{
Insulin Injections and Exercise Scheduling for Diabetics: An Optimal Control Model
}

\author{
Zahra Al Helal, Volker Rehbock*and Ryan Loxton \\ Department of Mathematics and Statistics, Curtin University, GPO Box U1987, Perth, WA 6845, Australia
}

\begin{abstract}
SUMMARY
The aim of this paper is to promote the development of new biological models as well as the application of optimization methods to these models. Fast and cheap computing power allows for the ready implementation of increasingly complex dynamic models in biology. However, these models are normally developed in isolation and their highly coupled nature can make it difficult to incorporate features from one model into another. In addition, there are many recent advances in numerical optimal control which have not yet been applied to biological models. In this paper, we illustrate how an existing biological model can be extended to incorporate features from other models, and we demonstrate that numerical optimal control techniques can readily determine optimal strategies for managing the resulting system. In particular, we develop a new composite dynamic model for the blood glucose regulatory system by incorporating the effects of exercise and insulin injections into an existing model with eight state variables. We formulate an optimal control problem in which the aim is to determine optimal injection times, optimal injection volumes and an optimal exercise regime to regulate the blood glucose level. A numerical approach, based on the concept of control parameterization and a time scaling transformation, is then developed for solving the optimal control problem. Numerical results for five scenarios show that optimal treatment regimes can be readily determined via the proposed approach. Copyright (C) 2017 John Wiley \& Sons, Ltd.
\end{abstract}

Received ...

KEY WORDS: Blood glucose; Optimal control; Diabetes; Control parameterization; Time scaling transformation

\section{INTRODUCTION}

A range of mathematical models have been proposed in the literature to capture and, in many cases, control blood glucose dynamics in the human body. Most of these models are dynamic in nature, taking the form of differential or difference equations. They typically incorporate other compounds associated with glucose, such as insulin, glucagon and glycogen. Some models are designed exclusively to determine treatment regimes for type 1 diabetics while others are intended for both healthy and diabetic individuals. Models also differ in terms of the processes that they capture. For example, some models simply assume the presence of glucose in the blood while others actually capture the digestive process directly. Models vary greatly in terms of their complexity, ranging from simple linear models involving just glucose and insulin [1] to complex nonlinear models that try to capture the chemical changes of the beta cells in the pancreas [2]. Although simple linear models are suitable for theoretical analysis and the application of standard control algorithms from the engineering disciplines, they do not capture the rich dynamic behavior of the real process. There is a range of medical treatment options for diabetes beyond the traditional use

${ }^{*}$ Correspondence to: Department of Mathematics and Statistics, Curtin University, GPO Box U1987, Perth, WA 6845, Australia. 
of insulin [3]. To capture the various effects of these treatments, mathematical models must include those dynamics that are directly affected by the treatments.

The Bergman minimal model [4] was proposed around 1980 to allow researchers to measure the quantitative contributions of pancreatic responsiveness (i.e., the increased production of insulin by the pancreas) and insulin sensitivity (i.e., the increased uptake of glucose by cells in response to insulin) to a subject's overall glucose tolerance (i.e., the body's ability to revert from high blood glucose levels back to base levels).

A more comprehensive model of the blood glucose regulatory system, which considers glucagon dynamics alongside insulin dynamics and their interaction, was proposed in [5]. This model involves three subsystems. The glucose subsystem is described by a one-compartment model of distribution and metabolism that involves net hepatic glucose balance (i.e., the difference between liver glucose production and uptake), renal excretion of glucose, insulin-dependent glucose utilization (by muscle) and insulin-independent glucose utilization (by the central nervous system). The insulin subsystem is described by a five-compartment model that involves pancreatic insulin storage, liver and portal plasma insulin, plasma insulin and insulin in the interstitial fluid. The glucagon subsystem is described by a one-compartment model that involves plasma glucagon and glucagon in the interstitial fluid.

The authors of [5] acknowledge the difficulty of validating complex models against experimental data. However, they point out that many aspects of their model are based on knowledge obtained from both whole body and individual organ experiments. Reference [5] also includes a range of simulation results for the model under various scenarios and reasonable results are obtained in each case. While acknowledging some shortcomings of their model, the authors of [5] make an important point that pertains to any comprehensive mathematical model in the biological sciences: 'The usefulness of the model as a sort of efficient and integrated library of physiological and clinical knowledge in a research group working both experimentally and theoretically on carbohydrate metabolism cannot be overemphasized'.

There were several publications around 1990 that focused on deriving optimal strategies for the control of diabetes. Both analytical and numerical solution methods were employed, including the first ever application of computational optimal control methods in this area [6]. In [6], the simple linear dynamic model of [1] was employed to describe the glucose dynamics while [7] makes use of the nonlinear Bergman minimal model [4]. The scenarios tested were based on a type 1 diabetic subject; the objectives were to reduce an initially high blood glucose level in one case, and to deal with a glucose spike due to a meal ingested in another case. The only means of controlling blood glucose was assumed to be the external administration of insulin. Some interesting results were obtained, such as a single large dose of insulin being superior to continuous injection, but the underlying models were too simple to justify any conclusions in a realistic environment.

In this work, we extend a mathematical model of the human blood glucose regulatory system originally proposed by Liu and Tang [8] and refined in [9]. Specifically, we focus on creating a more complete model that captures the effects of exercise and subcutaneous insulin injections on the blood glucose level for diabetic individuals. Based on the composite model, we formulate an optimal control problem that seeks to minimize the difference between the blood glucose level for a diabetic individual and a desired trajectory. To generate accurate values for the optimal insulin injection times, we apply a time scaling transformation (see [10] and [11]). Numerical results show that, on the basis of our optimal control model, good blood glucose control can be readily achieved for a variety of desired blood glucose trajectories with the use of the MISER3.3 optimal control software [12].

This paper is organized as follows. We first review the model described in [8] and [9] in Section 2. In Section 3, we propose extensions to the model that take into account the effects of both insulin injections and exercise. In Section 4, we formulate an optimal control problem with the aim of following a desired blood glucose profile as closely as possible. To allow for the numerical optimization of the injection times, we adopt the control parameterization and time scaling transformation methods. We then solve the transformed problem for a variety of scenarios 
using the optimal control software MISER3.3 in Section 5. Finally, we conclude the paper with a discussion of the numerical results and suggestions for future work.

\section{BLOOD GLUCOSE REGULATORY MODEL}

The dynamic model in Liu and Tang [8] involves eight state variables and can be naturally divided into three subsystems (see [8] and [9] for more details).

The insulin and glucagon transition subsystem governs the state variables $x_{1}$ and $x_{2}$, which represent the plasma concentrations of glucagon and insulin, respectively, both measured in moles per liter. The dynamics for $x_{1}$ are given by

$$
\frac{d x_{1}}{d t}=-\left(k_{1,1}^{p}+k_{1,2}^{p}\right) x_{1}+w_{1}
$$

where $k_{1,1}^{p}$ is a transition rate, $k_{1,2}^{p}$ is a degradation rate, and $w_{1}$ is the glucagon release rate (GRR) defined by

$$
w_{1}=\frac{G_{m}}{1+b_{1} \exp \left(a_{1}\left(x_{8}-C_{5}\right)\right)} .
$$

The dynamics for $x_{2}$ are given by

$$
\frac{d x_{2}}{d t}=-\left(k_{2,1}^{p}+k_{2,2}^{p}\right) x_{2}+w_{2},
$$

where $k_{2,1}^{p}$ is a transition rate, $k_{2,2}^{p}$ is a degradation rate, and $w_{2}$ is the insulin release rate (IRR) defined by

$$
w_{2}=\frac{R_{m}}{1+b_{2} \exp \left(a_{2}\left(C_{1}-x_{8}\right)\right)} .
$$

Variables $w_{1}$ and $w_{2}$ in equations (1)-(4) model the natural feedback control mechanisms in the body. Note that $G_{m}$ is the maximum glucagon release rate, $R_{m}$ is the maximum insulin infusion rate, and $a_{1}, a_{2}, b_{1}, b_{2}, C_{1}$ and $C_{5}$ are positive constants.

The insulin and glucagon receptor binding subsystem governs state variables $x_{3}, x_{4}, x_{5}$ and $x_{6}$. Variables $x_{3}$ and $x_{4}$ are the intracellular concentrations of glucagon and insulin, respectively, and $x_{5}$ and $x_{6}$ are the concentrations of glucagon and insulin bound receptors, respectively. As with $x_{1}$ and $x_{2}$, these states are measured in moles per liter. The dynamics for this subsystem are given by

$$
\begin{aligned}
& \frac{d x_{3}}{d t}=-k_{1,1}^{s} x_{3}\left(R_{1}^{0}-x_{5}\right)-k_{1,2}^{s} x_{3}+\frac{k_{1,1}^{p} V_{p} x_{1}}{V}, \\
& \frac{d x_{4}}{d t}=-k_{2,1}^{s} x_{4}\left(R_{2}^{0}-x_{6}\right)-k_{2,2}^{s} x_{4}+\frac{k_{2,1}^{p} V_{p} x_{2}}{V}, \\
& \frac{d x_{5}}{d t}=-k_{1,1}^{s} x_{3}\left(R_{1}^{0}-x_{5}\right)-k_{1}^{r} x_{5}, \\
& \frac{d x_{6}}{d t}=-k_{2,1}^{s} x_{4}\left(R_{2}^{0}-x_{6}\right)-k_{2}^{r} x_{6},
\end{aligned}
$$

where $k_{1,1}^{s}$ and $k_{2,1}^{s}$ are the hormone-receptor association rates, $k_{1,2}^{s}$ and $k_{2,2}^{s}$ are the degradation rates, $R_{1}^{0}$ and $R_{2}^{0}$ are the total concentrations of receptors, $k_{1}^{r}$ and $k_{2}^{r}$ are the inactivation rates, $V_{p}$ is the plasma insulin volume, and $V$ is the cellular insulin volume.

Finally, the glucose production and utilization subsystem governs state variables $x_{7}$ and $x_{8}$, which are the blood concentrations of glycogen and glucose, respectively, both measured in milligrams per liter. The dynamics for $x_{7}$ and $x_{8}$ are given by

$$
\frac{d x_{7}}{d t}=f_{4}-f_{5}
$$




\begin{tabular}{ccccccccccccc}
\hline$t$ & 0 & 60 & 90 & 120 & 150 & 180 & 240 & 300 & 360 & 420 & 480 & 540 \\
\hline$G$ & 0 & 69.60 & 69.28 & 77.46 & 83.73 & 85.75 & 89.24 & 87.14 & 72.99 & 52.74 & 37.61 & 30.30 \\
\hline
\end{tabular}

Table I. Parameter values for the exogenous glucose input rate $G$

and

$$
\frac{d x_{8}}{d t}=-f_{4}+f_{5}-f_{1}-f_{2} f_{3}+G
$$

where

$$
\begin{aligned}
& f_{1}=U_{b}\left(1-\exp \left(-\frac{x_{8}}{C_{2}}\right)\right), \\
& f_{2}=\frac{x_{8}}{C_{3}} \\
& f_{3}=U_{0}+\frac{\left(U_{m}-U_{0}\right) x_{4}^{\beta}}{C_{4}^{\beta}+x_{4}^{\beta}}, \\
& f_{4}=\frac{k_{1} x_{6}}{1+k_{2} x_{5}} \cdot \frac{V_{\max }^{g s} x_{8}}{k_{m}^{g s}+x_{8}}, \\
& f_{5}=k_{3} x_{5} \frac{V_{\max }^{g p} x_{7}}{k_{m}^{g p}+x_{7}} .
\end{aligned}
$$

Here, $f_{1}$ is the insulin-independent glucose utilization, the product of $f_{2}$ and $f_{3}$ models the insulin-dependent glucose utilization, $f_{4}$ represents the glycogen synthase, and $f_{5}$ is the glycogen phosphorylase. Furthermore, $U_{0}, U_{b}, U_{m}, C_{2}, C_{3}, C_{4}$, and $\beta$ are positive constants, $k_{1}, k_{2}$ and $k_{3}$ are the feedback control gains, $V_{\max }^{g s}$ is the maximum velocity of glycogen phosphorylase, $V_{\max }^{g p}$ is the maximum velocity of glycogen synthase, and $k_{m}^{g s}$ and $k_{m}^{g p}$ are Michaelis-Menten constants. Finally, for $G$ we use the exogenous glucose input rate from the experimental data published in Korach-André et al. [13] which is given in Table I. We assume a piecewise linear interpolation of this data as illustrated in Figure 1. This data represents a typical response to a meal, but note that such a response can vary greatly between subjects and also depends on the type of meal consumed.

As in [8], we assume that the system is modelled over a 9 hour period, i.e. $t \in[0,540]$, where $t$ is the time in minutes. The initial conditions prescribed for the model are

$$
\begin{aligned}
& x_{1}(0)=1.4 \times 10^{-11}, x_{2}(0)=1.389 \times 10^{-11}, x_{3}(0)=0, x_{4}(0)=6.945 \times 10^{-14}, \\
& x_{5}(0)=0, x_{6}(0)=0, x_{7}(0)=200, x_{8}(0)=918 .
\end{aligned}
$$

The complete model defined by equations (1)-(16) includes 36 model constants. In [8], the model was shown to follow the blood glucose profile of a healthy individual reasonably well when a particular set of values is chosen for the model constants. The blood glucose values were also taken from Korach-André et al. [13] and are listed in Table II. In [9], to further optimize the model constants, we formulated an optimal parameter selection problem in which the parameters are to be chosen so that the resulting model output best matches a piecewise linear interpolation of the blood glucose data in Table II. By solving this optimal parameter selection problem with the MISER3.3 optimal control software [12], a much closer match to the experimental data was achieved and the resulting optimized values of the model constants are listed in Tables III, IV, and V. The parameter estimation procedure used in MISER3.3 is a deterministic gradient based method which assumes that the data points in Table II are exact. Methods which take stochastic uncertainty of data into account are available [14], but were not used as there was no information regarding the measurement accuracy in [13].

We use the model parameter values in Tables III, IV, and V for the remainder of this paper. 


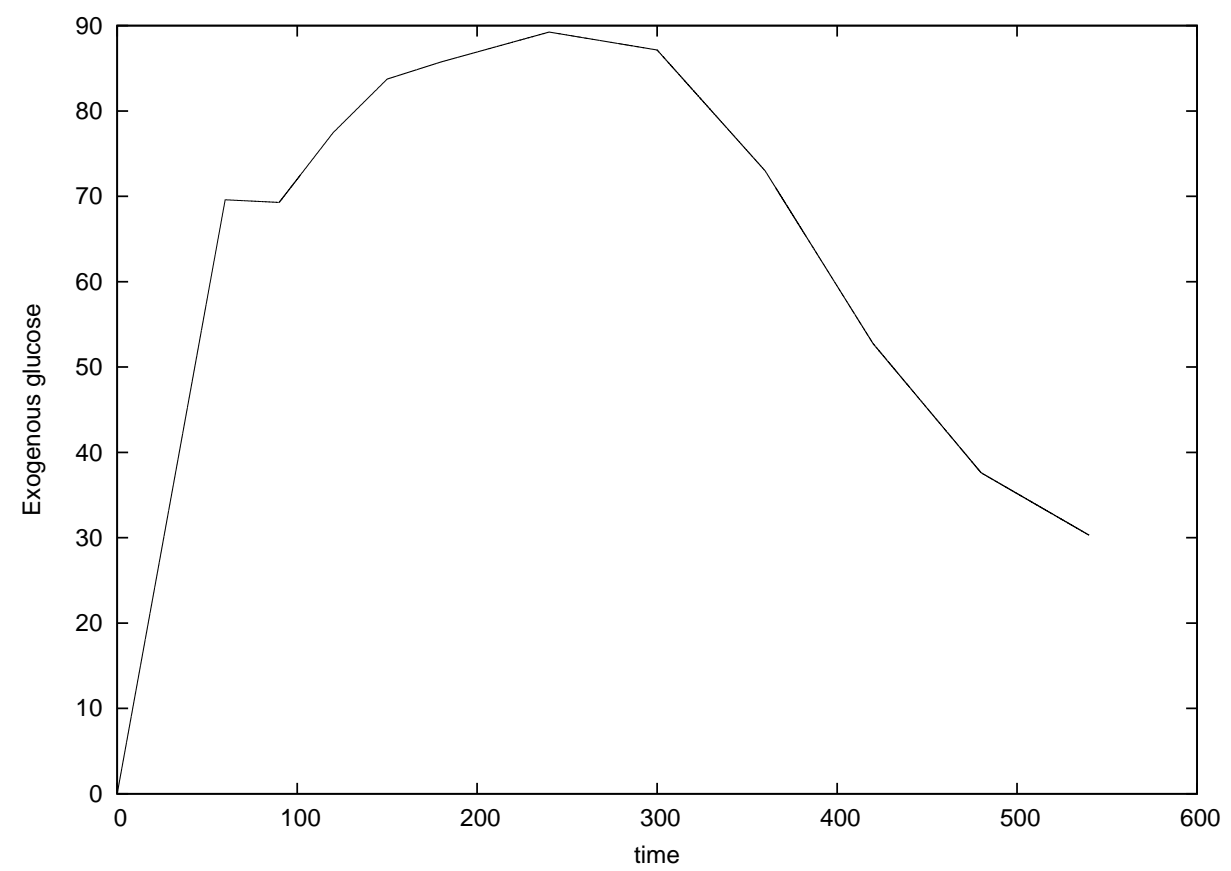

Figure 1. Exogenous glucose input rate from the experimental data of Korach-André et al. [13]

\begin{tabular}{cccccccccc}
\hline$i$ & 0 & 1 & 2 & 3 & 4 & 5 & 6 & 7 & 8 \\
\hline$\eta_{i}$ & 0 & 60 & 120 & 150 & 180 & 240 & 380 & 420 & 540 \\
\hline$\hat{x}_{8}^{i}$ & 900 & 1785.29 & 1530.27 & 1330.88 & 1300.55 & 1244.95 & 1113.53 & 1078.0 & 900.72 \\
\hline
\end{tabular}

Table II. The blood glucose levels at sample times $\eta_{i}, i=0, \ldots, 8$, from Korach-André et al. [13]

\begin{tabular}{ccc}
\hline Constant & Value & Unit \\
\hline$k_{1,1}^{p}$ & 1.28929 & $\mathrm{~min}^{-1}$ \\
\hline$k_{2,1}^{p}$ & 0.100804 & $\mathrm{~min}^{-1}$ \\
\hline$k_{1,2}^{p}$ & 0.3 & $\mathrm{~min}^{-1}$ \\
\hline$k_{2,2}^{p}$ & 0.437605 & $\mathrm{~min}^{-1}$ \\
\hline$G_{m}$ & $2.05367 \times 10^{-9}$ & $\mathrm{M} / \mathrm{min}$ \\
\hline$R_{m}$ & $2.29663 \times 10^{-10}$ & $\mathrm{M} / \mathrm{min}$ \\
\hline$C_{1}$ & $1.11419 \times 10^{3}$ & $\mathrm{mg} / 1$ \\
\hline$C_{5}$ & 1124.67 & $\mathrm{mg} / 1$ \\
\hline$a_{1}$ & 0.0348467 & $(\mathrm{mg} / 1)^{-1}$ \\
\hline$a_{2}$ & 0.0145946 & $(\mathrm{mg} / \mathrm{l})^{-1}$ \\
\hline$b_{1}$ & 11.471 & - \\
\hline$b_{2}$ & 1.15002 & - \\
\hline
\end{tabular}

Table III. Subsystem 1 parameters 


\begin{tabular}{ccc}
\hline Constant & Value & Unit \\
\hline$k_{1,1}^{s}$ & $6 \times 10^{7}$ & $\mathrm{M}^{-1} \mathrm{~min}^{-1}$ \\
\hline$k_{2,1}^{s}$ & $6 \times 10^{7}$ & $\mathrm{M}^{-1} \mathrm{~min}^{-1}$ \\
\hline$k_{1,2}^{s}$ & $7.19985 \times 10^{-3}$ & $\mathrm{~min}^{-1}$ \\
\hline$k_{2,2}^{s}$ & $9.52782 \times 10^{-3}$ & $\mathrm{~min}^{-1}$ \\
\hline$k_{1}^{r}$ & $2.59194 \times 10^{-2}$ & $\mathrm{~min}^{-1}$ \\
\hline$k_{1,1}^{p}$ & 1.28929 & $\mathrm{~min}^{-1}$ \\
\hline$k_{2,1}^{p}$ & 0.100804 & $\mathrm{~min}^{-1}$ \\
\hline$k_{2}^{r}$ & 0.2 & $\mathrm{~min}^{-1}$ \\
\hline$R_{1}^{0}$ & $9 \times 10^{-13}$ & $\mathrm{M}$ \\
\hline$R_{2}^{0}$ & $3.6114 \times 10^{-12}$ & $\mathrm{M}$ \\
\hline$V$ & 10.0004 & 1 \\
\hline$V_{p}$ & 2.41375 & 1 \\
\hline
\end{tabular}

Table IV. Subsystem 2 parameters

\begin{tabular}{ccc}
\hline Constant & Value & Unit \\
\hline$V_{\max }^{g p}$ & 25.0197 & $\mathrm{mg} / \mathrm{l} / \mathrm{min}$ \\
\hline$k_{m}^{g p}$ & 600 & $\mathrm{mg} / \mathrm{l}$ \\
\hline$V_{\max }^{g s}$ & $3.41805 \times 10^{-3}$ & $\mathrm{mg} / \mathrm{l} / \mathrm{min}$ \\
\hline$k_{m}^{g s}$ & 67 & $\mathrm{mg} / \mathrm{l}$ \\
\hline$k_{1}$ & $2.76901 \times 10^{11}$ & $\mathrm{M}^{-1}$ \\
\hline$k_{2}$ & $1.1111111 \times 10^{14}$ & $\mathrm{M}^{-1}$ \\
\hline$k_{3}$ & $1.1111111 \times 10^{12}$ & $\mathrm{M}^{-1}$ \\
\hline$U_{b}$ & 4 & $\mathrm{mg} / \mathrm{l} / \mathrm{min}$ \\
\hline$U_{0}$ & 4 & $\mathrm{mg} / \mathrm{l} / \mathrm{min}$ \\
\hline$U_{m}$ & 227.508 & $\mathrm{mg} / \mathrm{l} / \mathrm{min}$ \\
\hline$C_{2}$ & 345.384 & $\mathrm{mg} / \mathrm{l}$ \\
\hline$C_{3}$ & 1061.82 & $\mathrm{mg} / \mathrm{l}$ \\
\hline$C_{4}$ & $1.9556 \times 10^{-9}$ & $\mathrm{M} / \mathrm{l}$ \\
\hline$\beta$ & 1.14055 & - \\
\hline
\end{tabular}

Table V. Subsystem 3 parameters

\section{NEW COMPOSITE MODEL WITH EXERCISE AND INSULIN INJECTIONS}

To adapt the Liu and Tang model to a diabetic subject, we must assume that the natural insulin release rate defined by equation (4) is impaired. For a type 1 diabetic, the maximum insulin release rate should be chosen as $R_{m}=0$. For a type 2 diabetic, we should choose $R_{m} \in(0,1)$ depending on the severity of the condition. 


\begin{tabular}{ccccc}
\hline \multirow{2}{*}{ Insulin type } & \multicolumn{4}{c}{ Parameter } \\
\cline { 2 - 5 } & $s$ & $a$ & $b$ & $V_{d}$ \\
\hline Short acting (soluble) & 2 & $4.31965 \times 10^{5}$ & 102 & $12 \times 10^{-6}$ \\
\hline Intermediate acting (NPH) & 2 & $1.5550756 \times 10^{6}$ & 294 & $12 \times 10^{-6}$ \\
\hline Long acting (ultralente) & 2.5 & 0 & 780 & $12 \times 10^{-6}$ \\
\hline Unit & - & $\mathrm{min} / \mathrm{M}$ & $\mathrm{min}$ & $\mathrm{L}$ \\
\hline
\end{tabular}

Table VI. Berger model parameters for different types of insulin; intermediate, short and long acting insulin

\subsection{SC injections}

A variety of mathematical models for the absorption of insulin from subcutaneous (SC) injections into blood plasma have been proposed in the literature. The complexity of these models varies significantly, starting with a simple single pool model involving a delay term to approximate the absorption of fast acting insulin [15]. Trajanski et al. [16] present a far more complex model where the diffusion of insulin from the injection site is approximated via the transition across a series of concentric spherical shells. This leads to a series of partial differential equations and, while accurate results can certainly be achieved, there is a significant computational cost associated with this model. A detailed review of various absorption models is given in [17]. After careful consideration of the suitability of each of these models for our purposes and, guided by the conclusions in [17], we settled on the model first proposed in Berger et al. [18]. Briefly, in this model the amount of absorbed insulin from the $\mathrm{SC}$ injection depot, $A(t)$, is assumed to be given by

$$
A(t)=1-\frac{t^{s}}{\left(T_{50}\right)^{s}+t^{s}},
$$

where $t$ is the time elapsed since the injection, $s$ is a constant depending on the insulin type and $T_{50}$ is the duration required to reach a $50 \%$ absorption of the injected insulin. Note that $T_{50}$ is given by

$$
T_{50}=a D+b,
$$

where $D$ is the insulin dose and $a$ and $b$ are constants which assume different values for different insulin preparations. Table VI shows these parameter values for different types of insulin. The time derivative of $A(t)$, multiplied by the injection dose, then gives the input flux of injected insulin into the plasma,

$$
\frac{t^{s-1} s\left(T_{50}\right)^{s} D}{V_{d}\left(\left(T_{50}\right)^{s}+t^{s}\right)^{2}}
$$

where $V_{d}$ is the plasma insulin distribution volume.

We now allow for up to $M+1 \mathrm{SC}$ insulin injections over the time horizon $[0, T]$. Each one of these has an individual effect on the plasma glucose concentration and this is modelled by replacing equation (3) with

$$
\frac{d x_{2}}{d t}=-\left(k_{2,1}^{p}+k_{2,2}^{p}\right) x_{2}+w_{2}+\sum_{i=0}^{M} I_{i}(t),
$$

where

$$
I_{i}(t)=\frac{s_{i}\left(t-t_{i}\right)^{s_{i}-1}\left(T_{i}\right)^{s_{i}} D_{i} H\left(t-t_{i}\right)}{V_{d}\left(\left(T_{i}\right)^{s_{i}}+\left(t-t_{i}\right)^{s_{i}}\right)^{2}}, \quad i=0,1, \ldots, M,
$$

and $t_{i}, i=0,1, \ldots, M$, are the insulin injection times with $t_{0}=0$ (we assume an injection at the initial time) and $H$ is the Heaviside step function defined by

$$
H(t)= \begin{cases}0, & \text { if } t<0 \\ 1, & \text { if } t \geq 0\end{cases}
$$


Furthermore, $T_{i}, i=0,1, \ldots, M$, are the durations needed for a $50 \%$ absorption of the insulin injected at time $t_{i}$, defined by

$$
T_{i}=a_{i} D_{i}+b_{i}, i=0,1, \ldots, M,
$$

where $D_{i}$, measured in moles (M), is the insulin dose and $a_{i}, b_{i}$ and $s_{i}$ are specific to the type of insulin preparation administered at time $t_{i}$. We assume that both the timing of each injection, $t_{i}$, and the corresponding dosage, $D_{i}, i=0,1, \ldots, M$, are variables to be determined while the insulin type (i.e., the choice of each $a_{i}, b_{i}$ and $s_{i}$ ) is specified by the user. Note that the dosage for each injection is bounded.

\subsection{Exercise modelling}

It is well known that physical exercise has a significant effect on blood glucose levels. Indeed, one of the common ways to deal with mild cases of type 2 diabetes is to prescribe increased exercise for patients along with sensible changes in diet. It is thus surprising to see that attempts to incorporate the effects of exercise into glucose-insulin dynamic models have only appeared in the literature relatively recently. This, together with the fact that most of the existing approaches have little to no overlap, seems to indicate that the task is not an easy one and that there are likely to be multiple pathways through which exercise impacts both glucose and insulin levels in the blood. In [19], the impact of exercise is modelled rather simply by perturbing coefficients in the well known Bergman minimal model [4]. The main aim in [19] is to study the stability properties of equilibria for different scenarios. Reference [20] follows a similar approach and also introduces some additional dynamic states (energy consumption and insulin action). The main focus of [20] was to fit the resulting model to the results from a clinical study. The authors of [21] start with a different glucose-insulin model [22] and emphasize the importance of the redistribution of blood flows around the body during periods of exercise. None of the models mentioned are suitable for incorporation with the model described in Section 2, as they are all based on much simpler glucose-insulin models involving only 3 state variables. Reference [23] introduces a much more comprehensive model. This takes into account the important role of free fatty acids (FFAs) in the blood stream as a source of energy for the body. It is argued that there are important interactions between the levels of FFAs, insulin and exercise that have not been accounted for in previous models. While we are not incorporating the complete model from [23] into the one from Section 2 (this would be an interesting challenge for future studies), we are adopting several aspects related to the impact of exercise. First, it is argued that exercise promotes a clearance of insulin in the blood stream since this results in higher glucose production in the liver, which is needed to provide energy. Mathematically, this is modelled as a second order effect with the dynamics

$$
\begin{aligned}
\frac{d x_{9}}{d t} & =m_{p v}\left(u_{e x}-x_{9}\right) \\
\frac{d x_{10}}{d t} & =m_{I U 1} x_{9}-m_{I U 2} x_{10}
\end{aligned}
$$

where $x_{9} \in[0,100]$ represents the current percentage of the maximum rate of oxygen consumption for an individual (which, in turn, is assumed to be linearly proportional to the energy expenditure) and $x_{10}$ is the rate at which insulin is cleared from the blood due to this effect. Here, $u_{e x} \in[0,100]$ is the rate of oxygen consumption due to exercise (itself measured as a percentage of the maximum rate of oxygen consumption for an individual). Moreover, $m_{p v}, m_{I U 1}$ and $m_{I U 2}$ are model constants whose values are listed in Table VII. Except for $m_{I U 1}$, these tabulated values are adopted from [23]. For $m_{I U 1}$, we reduced the value reported in [23] by a factor 10, as the original value appeared to result in the blood insulin level decreasing far more quickly than the rate shown by experimental data in [23]. Finally, equation (20) is modified as follows

$$
\frac{d x_{2}}{d t}=-\left(k_{2,1}^{p}+k_{2,2}^{p}\right) x_{2}+w_{2}+\sum_{i=0}^{M} I_{i}-x_{10} x_{2} .
$$




\begin{tabular}{ccc}
\hline Constant & Value & Unit \\
\hline$m_{p v}$ & 0.8 & $\min ^{-1}$ \\
\hline$m_{I U 1}$ & $2.8176 \times 10^{-4}$ & $\min ^{-2}$ \\
\hline$m_{I U 2}$ & 1.7354 & $\mathrm{~min}^{-1}$ \\
\hline$m_{G U 1}$ & $2.1874 \times 10^{-3}$ & $\mathrm{mg} /\left(\mathrm{kg} \times \mathrm{min}^{2}\right)$ \\
\hline$m_{G U 2}$ & $5.8974 \times 10^{-2}$ & $\mathrm{~min}^{-1}$ \\
\hline$m_{G P 1}$ & $9.152 \times 10^{-4}$ & $\mathrm{mg} /\left(\mathrm{kg} \times \mathrm{min}^{2}\right)$ \\
\hline$m_{G P 2}$ & 1.3073 & $\mathrm{~min}^{-1}$ \\
\hline$W$ & 70 & $\mathrm{~kg}$ \\
\hline$V_{G}$ & 11.7 & 1
\end{tabular}

Table VII. Parameter values related to exercise effects on glucose and insulin dynamics

The authors of [23] also indicate that exercise induces an increase in the glucose uptake rate by the working muscles. Furthermore, the rate of glucose production is also increased due to an accelerated rate of glycogenolysis (conversion of glycogen into glucose-6-phosphate, which is further converted to glucose in the liver). These effects are modelled by the following dynamics,

$$
\frac{d x_{11}}{d t}=m_{G U 1} x_{9}-m_{G U 2} x_{11}
$$

$$
\frac{d x_{12}}{d t}=m_{G P 1} x_{9}-m_{G P 2} x_{12},
$$

where $x_{11}$ and $x_{12}$ represent the exercise induced glucose uptake and production rates, respectively. Furthermore, $m_{G U 1}, m_{G U 2}, m_{G P 1}$ and $m_{G P 2}$ are constants whose values are adopted from [23] and also listed in Table VII. Finally, the effects are incorporated into blood glucose dynamic equation (10) as follows:

$$
\frac{d x_{8}}{d t}=-f_{4}+f_{5}-f_{1}-f_{2} f_{3}+G+\frac{W}{V_{G}}\left(x_{12}-x_{11}\right)
$$

where $W$ and $V_{G}$ are model constants whose values are also taken from [23] and given in Table VII. 


\subsection{Summary of the revised model}

In summary, the revised dynamical model is described as follows:

$$
\begin{aligned}
\frac{d x_{1}}{d t} & =-\left(k_{1,1}^{p}+k_{1,2}^{p}\right) x_{1}+w_{1}, \\
\frac{d x_{2}}{d t} & =-\left(k_{2,1}^{p}+k_{2,2}^{p}\right) x_{2}+w_{2}+\sum_{i=0}^{M} I_{i}-x_{10} x_{2}, \\
\frac{d x_{3}}{d t} & =-k_{1,1}^{s} x_{3}\left(R_{1}^{0}-x_{5}\right)-k_{1,2}^{s} x_{3}+\frac{k_{1,1}^{p} V_{p} x_{1}}{V}, \\
\frac{d x_{4}}{d t} & =-k_{2,1}^{s} x_{4}\left(R_{2}^{0}-x_{6}\right)-k_{2,2}^{s} x_{4}+\frac{k_{2,1}^{p} V_{p} x_{2}}{V}, \\
\frac{d x_{5}}{d t} & =-k_{1,1}^{s} x_{3}\left(R_{1}^{0}-x_{5}\right)-k_{1}^{r} x_{5}, \\
\frac{d x_{6}}{d t} & =-k_{2,1}^{s} x_{4}\left(R_{2}^{0}-x_{6}\right)-k_{2}^{r} x_{6}, \\
\frac{d x_{7}}{d t} & =f_{4}-f_{5}, \\
\frac{d x_{8}}{d t} & =-f_{4}+f_{5}-f_{1}-f_{2} f_{3}+G+\frac{W}{V_{G}}\left(x_{12}-x_{11}\right), \\
\frac{d x_{9}}{d t} & =m_{p v}\left(u_{E x}-x_{9}\right), \\
\frac{d x_{10}}{d t} & =m_{I U 1} x_{9}-m_{I U 2} x_{10}, \\
\frac{d x_{11}}{d t} & =m_{G U 1} x_{9}-m_{G U 2} x_{11}, \\
\frac{d x_{12}}{d t} & =m_{G P 1} x_{9}-m_{G P 2} x_{12},
\end{aligned}
$$

where $I_{i}, i=0, \ldots, M$, are defined by (21). System (30)-(41) is subject to the initial conditions

$$
\begin{aligned}
& x_{1}(0)=1.4 \times 10^{-11}, x_{2}(0)=1.389 \times 10^{-11}, x_{3}(0)=0, x_{4}(0)=6.945 \times 10^{-14}, \\
& x_{5}(0)=0, x_{6}(0)=0, x_{7}(0)=200, x_{8}(0)=918, \\
& x_{9}(0)=0, x_{10}(0)=0, x_{11}(0)=0, x_{12}(0)=0 .
\end{aligned}
$$

The complete composite model defined by equations (30)-(42) includes the model constants listed in Tables III-VII. We again assume that the system is modelled over a 9 hour period, i.e., $t \in[0,540]$, where $t$ is the time in minutes.

\section{OPTIMAL CONTROL PROBLEM}

\subsection{Problem statement}

We first formulate an optimal control problem based on the composite model (30)-(42) for a diabetic individual. Our aim is to match the subject's blood glucose level with a desired blood glucose profile $g_{d}(t)$ over the entire time horizon. Thus, we need to choose injection times $t_{i}, i=1, \ldots, M$, injection dosages $D_{i}, i=0,1, \ldots, M$, and an exercise regime $u_{e x}(t), t \in[0,540]$, with the aim to minimize

$$
J=\int_{0}^{540}\left(x_{8}(t)-g_{d}(t)\right)^{2} d t
$$

subject to the dynamics (30)-(42), the control bounds

$$
0 \leqslant u_{e x}(t) \leqslant 100, t \in[0,540],
$$


the bounds on the injection times

$$
0 \leqslant t_{i} \leqslant 540, i=1, \ldots, M
$$

and the dosage bounds

$$
0 \leqslant D_{i} \leqslant D_{i, \max }, i=0,1, \ldots, M
$$

\subsection{Control parameterization}

For computational purposes, we assume that $u_{e x}$ is a piecewise constant function defined over a partition $P=\left\{\tau_{0}, \tau_{1}, \ldots, \tau_{N}\right\}$ with $\tau_{0}=0, \tau_{N}=540$ and $\tau_{i-1} \leqslant \tau_{i}, i=1, \ldots, N$, where $N$ is the number of intervals in the partition chosen by the user. This is a commonly used control parameterization scheme (see references [10], [11] for details).

For convenience, we also assume that the insulin injection times $t_{i}, i=1, \ldots, M$, coincide with some of the switching times of $u_{e x}$, i.e.,

$$
t_{i}=\tau_{k_{i}}, i=1, \ldots, M
$$

where $k_{i} \in K=\left\{k_{1}, k_{2}, \ldots, k_{M}\right\} \subset\{0,1, \ldots, N\}$. Note that $t_{0}=0=\tau_{0}$. Naturally, we also assume that $N>>M$.

\subsection{Time scaling transformation}

As with most optimal control softwares employing the control parameterization technique MISER3.3 does not allow the time points $\tau_{i}, i=1, \ldots, N$, to be variable. In fact, there are serious numerical difficulties associated with optimizing variable switching times directly [10]. To overcome this hurdle, we invoke a well known transformation [10] to map the variable time points $\tau_{i}, i=1, \ldots, N$, to fixed points in a new time horizon $[0, N]$. This is achieved by defining a new time variable $s \in[0, N]$ and expressing the old time variable $t$ in terms of $s$ through the differential equation

$$
\frac{d t(s)}{d s}=v(s)
$$

where $v$ is a piecewise constant function with break points at $0,1,2, \ldots, N$, and subject to the lower and upper bounds

$$
0 \leqslant v(s) \leqslant 540, s \in[0, N]
$$

Furthermore, we require

$$
t(0)=\tau_{0}=0
$$

and

$$
t(N)=T=540 .
$$

Note that the values of $\tau_{i}, i=1, \ldots, N$, can be easily calculated once $v$ has been chosen, since each piecewise constant value of $v$ is equal to the difference between the corresponding successive switching times [10]. Note that the upper bound on $v$ in (49) is not unduly restrictive as the duration between successive switching times clearly cannot exceed the duration of the time horizon $T=540$.

We adopt the notation $\tilde{x}_{i}(s)=x_{i}(t(s)), i=1, \ldots, 12, \tilde{g}_{d}(s)=g_{d}(t(s)), \tilde{I}_{i}(s)=I_{i}(t(s)), \tilde{G}(s)=$ $G(t(s))$ and $\tilde{u}_{e x}(s)=u_{e x}(t(s))$, where $\tilde{u}_{e x}(s)$, like $v(s)$, is also assumed to be piecewise constant with break points at $\{0,1,2, \ldots, N\}$. The new control $\tilde{u}_{e x}(s)$ must satisfy the bound constraints

$$
0 \leqslant \tilde{u}_{e x}(s) \leqslant 100, s \in[0, N]
$$

Changing the time variable from $t$ to $s$ in (30)-(41) through the use of (48)-(51), we obtain the following dynamical system: 


$$
\begin{aligned}
\frac{d \tilde{x}_{1}}{d s} & =\left(-\left(k_{1,1}^{p}+k_{1,2}^{p}\right) \tilde{x}_{1}+w_{1}\right) v(s), \\
\frac{d \tilde{x}_{2}}{d s} & =\left(-\left(k_{2,1}^{p}+k_{2,2}^{p}\right) \tilde{x}_{2}+w_{2}+\sum_{i=1}^{M} \tilde{I}_{i}(s)-\tilde{x}_{10} \tilde{x}_{2}\right) v(s), \\
\frac{d \tilde{x}_{3}}{d s} & =\left(-k_{1,1}^{s} \tilde{x}_{3}\left(R_{1}^{0}-\tilde{x}_{5}\right)-k_{1,2}^{s} \tilde{x}_{3}+\frac{k_{1,1}^{p} V_{p} \tilde{x}_{1}}{V}\right) v(s), \\
\frac{d \tilde{x}_{4}}{d s} & =\left(-k_{2,1}^{s} \tilde{x}_{4}\left(R_{2}^{0}-\tilde{x}_{6}\right)-k_{2,2}^{s} \tilde{x}_{4}+\frac{k_{2,1}^{p} V_{p} \tilde{x}_{2}}{V}\right) v(s), \\
\frac{d \tilde{x}_{5}}{d s} & =\left(-k_{1,1}^{s} \tilde{x}_{3}\left(R_{1}^{0}-\tilde{x}_{5}\right)-k_{1}^{r} \tilde{x}_{5}\right) v(s), \\
\frac{d \tilde{x}_{6}}{d s} & =\left(-k_{2,1}^{s} \tilde{x}_{4}\left(R_{2}^{0}-\tilde{x}_{6}\right)-k_{2}^{r} \tilde{x}_{6}\right) v(s), \\
\frac{d \tilde{x}_{7}}{d s} & =\left(f_{4}-f_{5}\right) v(s), \\
\frac{d \tilde{x}_{8}}{d s} & =\left(-f_{4}+f_{5}-f_{1}-f_{2} f_{3}+\tilde{G}(s)+W\left(\tilde{x}_{12}-\tilde{x}_{11}\right)\right) v(s), \\
\frac{d \tilde{x}_{9}}{d s} & =m_{p v}\left(\tilde{u}_{e x}-\tilde{x}_{9}\right) v(s), \\
\frac{d \tilde{x}_{10}}{d s} & =\left(m_{I U 1} \tilde{x}_{9}-m_{I U 2} \tilde{x}_{10}\right) v(s), \\
\frac{d \tilde{x}_{11}}{d s} & =\left(m_{G U 1} \tilde{x}_{9}-m_{G U 2} \tilde{x}_{11}\right) v(s), \\
\frac{d \tilde{x}_{12}}{d s} & =\left(m_{G P 1} \tilde{x}_{9}-m_{G P 2} \tilde{x}_{12}\right) v(s), \\
\frac{d t(s)}{d s} & =v(s) . \\
& \left.=f_{2,}\right),(12),(13)
\end{aligned}
$$

Here, $w_{1}, w_{2}, f_{1}, f_{2}, f_{3}, f_{4}$ and $f_{5}$ are again defined by (2), (4), (11), (12), (13), (14) and (15), respectively, with $t$ replaced by $t(s)$.

The transformed problem may now be stated as: Choose $v(s), \tilde{u}_{e x}(s), t_{i}, i=1, \ldots, M$, and $D_{i}$, $i=0,1, \ldots, M$, to minimize

$$
\tilde{J}=\int_{0}^{N} v(s)\left(\tilde{x}_{8}(s)-\tilde{g}_{d}(s)\right)^{2} d s
$$

subject to the dynamics (53)-(65), the initial conditions (42) and (50), the control bounds (49) and (52) and the constraints

$$
g_{i}=t\left(k_{i}\right)-t_{i}=0, i=1, \ldots, M,
$$

and

$$
g_{M+1}=T-t(N)=0 .
$$

Constraint (67) ensures that the time scaling transformation maps $s=k_{i}$ to the $i$ th injection time as required in the control parameterization scheme discussed in Section 4.2. Similarly, constraint (68) ensures that $s=N$ is mapped to the terminal time.

There is one feature in the transformed problem that prevents it from being implemented directly in MISER3.3. In the transformed objective (66), we have the function $g_{d}(t(s))$, where $t(s)$ is a state variable in the transformed problem. MISER3.3 is based on the assumption that the objective integrand is differentiable with respect to the state variables, but choosing $g_{d}(t(s))=g_{\exp }(t(s))$ as defined in Section 2 will result in a piecewise linear function that is not differentiable with respect to $t$. Thus, we change $g_{\exp }$ from a piecewise linear form to a piecewise quadratic form defined in equation (69) below and illustrated in Figure 2:

$$
\tilde{g}_{d}=\alpha_{i} t(s)^{2}+\beta_{i} t(s)+\gamma_{i}, \quad t(s) \in\left[\eta_{i-1}, \eta_{i}\right], i=1, \ldots, 8,
$$




\begin{tabular}{ccccc}
\hline$i$ & $\alpha_{i}$ & $\beta_{i}$ & $\gamma_{i}$ & $\eta_{i}$ \\
\hline 1 & $-1.182857143 \times 10^{-3}$ & 0.1656 & 5 & 60 \\
\hline 2 & $-9.178 \times 10^{-4}$ & 0.128492 & 6.29878 & 120 \\
\hline 3 & $1.828555556 \times 10^{-3}$ & -0.5306333333 & 45.8463 & 150 \\
\hline 4 & $-7.85 \times 10^{-4}$ & 0.2534333333 & -12.9587 & 180 \\
\hline 5 & $4.003055556 \times 10^{-4}$ & -0.1732766667 & 25.4452 & 240 \\
\hline 6 & $-1.720357143 \times 10^{-4}$ & 0.1014471429 & -7.521657143 & 380 \\
\hline 7 & $6.098125 \times 10^{-4}$ & -0.4927575 & 105.3772250 & 420 \\
\hline 8 & $-2.308472222 \times 10^{-4}$ & 0.2133966667 & -42.91515 & 540 \\
\hline
\end{tabular}

Table VIII. Parameter values for the piecewise quadratic interpolation of the $g_{d}$ function

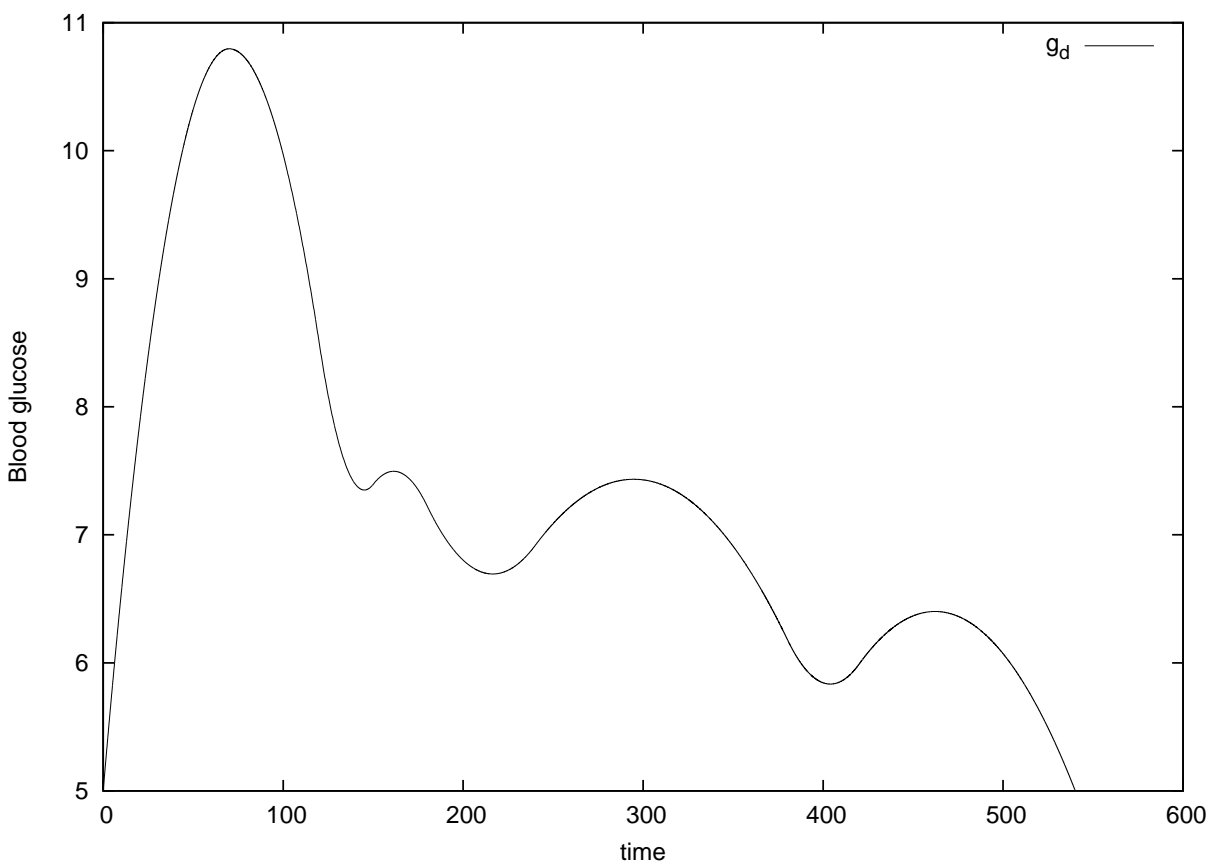

Figure 2. Piecewise quadratic interpolation of the experimental data

where $\eta_{0}, \eta_{1}, \ldots, \eta_{8}$ are as defined in Table II. The parameter values defining this function are determined from a smooth piecewise quadratic interpolation of the data in Table II and they are listed in Table VIII. In summary, the transformed dynamic model consists of 13 state variables, $\tilde{x}_{i}(s), i=1, \ldots, 12$ and $t(s), 2$ control functions, $\tilde{u}_{e x}(s)$ and $v(s)$, and $2 M+1$ system parameters, $D_{i}, i=0,1, \ldots, M$, and $t_{i}, i=1, \ldots, M$. These system parameters represent, respectively, the volume of insulin injections and time points of these injections. For the numerical results presented below, we assume up to 5 individual injections (i.e., $M=4$ ). We also assume $N=20$ and choose $k_{0}=0, k_{1}=4, k_{2}=8, k_{3}=12$ and $k_{4}=16$ for the constraints given in (67). This version of the problem is in a canonical form suitable for MISER3.3. Note that the graphical results in the next section are given in terms of the orginal time scale, $t \in[0,540]$. 


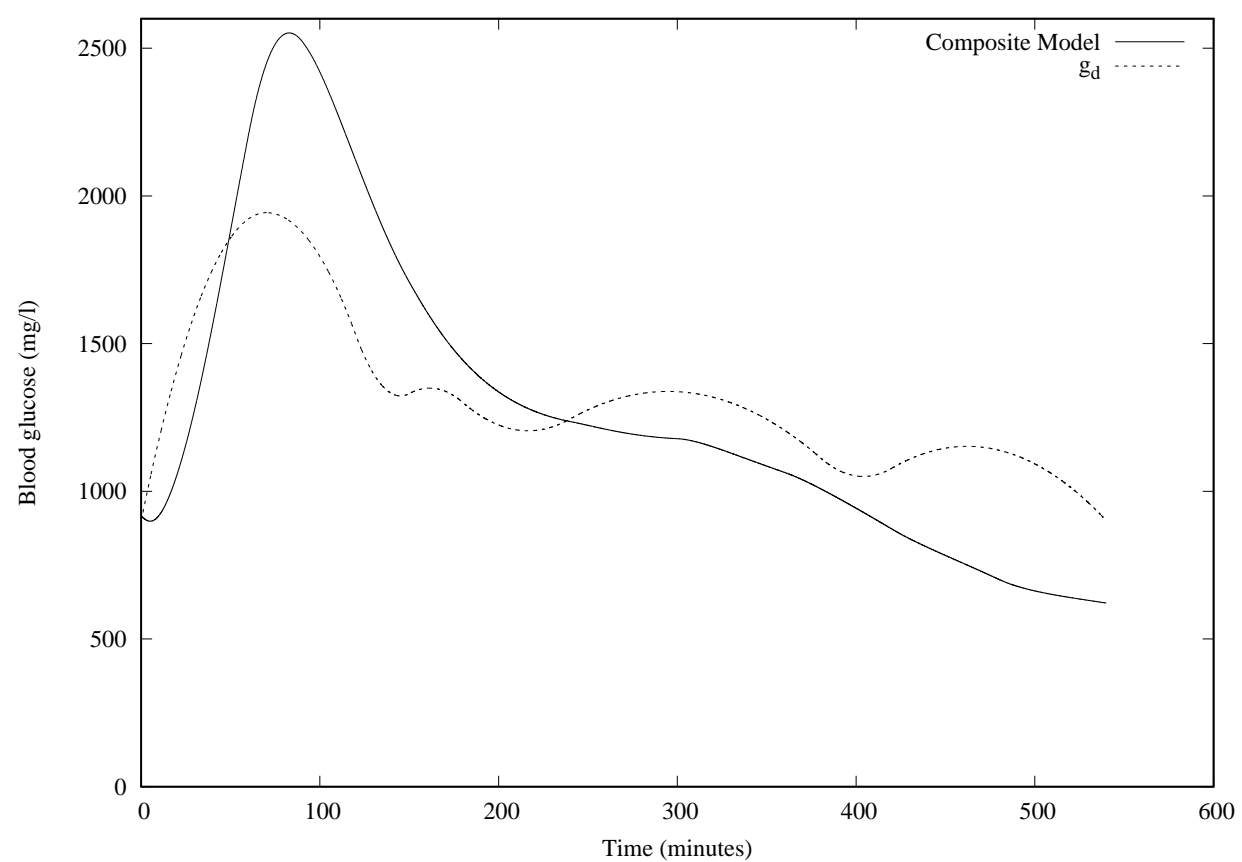

Figure 3. Comparison of two blood glucose trajectories: the solid curve is the optimal trajectory for Case 1; the dashed curve is the desired trajectory.

\section{NUMERICAL RESULTS}

\subsection{Case 1: NPH injection with no exercise}

The first case that we consider is for a type 1 diabetic $\left(R_{m}=0\right)$ with only one intermediate acting insulin $(\mathrm{NPH})$ injection at the beginning of the time horizon $\left(t_{0}=0\right)$ and with no exercise. Here, we assume that the desired blood glucose function is $g_{d}(t)=g_{\text {exp }}(t)$, where $g_{\text {exp }}$ is in piecewise quadratic form as shown in Figure 2. This is a relatively simple version of the problem with only one decision variable $\left(D_{0}\right)$, the dosage of insulin injected at $t_{0}=0$, where

$$
0 \leqslant D_{0} \leqslant 2.0835 \times 10^{-4}
$$

An optimal solution is readily obtained by MISER3.3. Figure 3 shows that the optimal blood glucose trajectory follows the desired blood glucose profile only briefly near the beginning of the time horizon. The optimal insulin injection dosage $D_{0}$ is at the upper bound $2.0835 \times 10^{-4} M$ which is equivalent to $30 U$. The optimal objective function value for this case is 1689 . Clearly, one injection is not enough to achieve good glucose control over the entire time horizon and we will need to consider multiple injections as well as exercise.

\subsection{Case 2: Transformed composite model without exercise}

This case also assumes a type 1 diabetic $\left(R_{m}=0\right)$ and we once again use $g_{d}(t)=g_{\text {exp }}(t)$. We consider two different types of insulin preparations, intermediate and short acting in combination. Details of the bounds and initial guesses of $t_{i}, i=1, \ldots, 5$, are given in Table IX. The bounds on the dosages are given in equation (71) below. Note that either intermediate or slow acting insulin may be chosen for each injection and corresponding values of $a_{i}, b_{i}$ and $s_{i}, i=0, \ldots, M$, must also be chosen. We indicate the chosen values in Table X. In this case, we assume that the first injection is 


\begin{tabular}{cccc}
\hline Injection time $t_{i}$ & Initial guess & Lower bound & Upper bound \\
\hline$t_{0}$ & 0 & 0 & 0 \\
\hline$t_{1}$ & 108 & 0 & 540 \\
\hline$t_{2}$ & 216 & 0 & 540 \\
\hline$t_{3}$ & 324 & 0 & 540 \\
\hline$t_{4}$ & 432 & 0 & 540 \\
\hline
\end{tabular}

Table IX. Decision variables $t_{i}, i=0, \ldots, 4$, for Cases $2-4$.

\begin{tabular}{cccc}
\hline \multirow{2}{*}{ Insulin dosage } & \multicolumn{3}{c}{ Parameter values } \\
\cline { 2 - 4 } & $s_{i}$ & $a_{i}$ & $b_{i}$ \\
\hline$D_{0}$ & 2 & $1.5550756 \times 10^{6}$ & 294 \\
\hline$D_{1}$ & 2 & $4.31965 \times 10^{5}$ & 102 \\
\hline$D_{2}$ & 2 & $1.5550756 \times 10^{6}$ & 294 \\
\hline$D_{3}$ & 2 & $4.31965 \times 10^{5}$ & 102 \\
\hline$D_{4}$ & 2 & $1.5550756 \times 10^{6}$ & 294 \\
\hline
\end{tabular}

Table X. Insulin dosages and the corresponding parameter values of $a_{i}, b_{i}$ and $s_{i}, i=0, \ldots, 4$, for Cases 2-4.

\begin{tabular}{cccc}
\hline Dosages $D_{i}$ & Optimal values & Times $t_{i}$ & Optimal values \\
\hline$D_{0}$ & $1.16761 \times 10^{-04} M \equiv 16.8 U$ & $t_{0}$ & 0 \\
\hline$D_{1}$ & $5.08103 \times 10^{-05} M \equiv 7.3 U$ & $t_{1}$ & 12.7618 \\
\hline$D_{2}$ & 0 & $t_{2}$ & 203.279 \\
\hline$D_{3}$ & 0 & $t_{3}$ & 327.564 \\
\hline$D_{4}$ & 0 & $t_{4}$ & 438.667 \\
\hline
\end{tabular}

Table XI. Optimal values of decision variables $D_{i}$ and $t_{i}$ in Case 2.

an intermediate acting insulin while subsequent injections alternate between short and intermediate acting. The dosage bounds are

$$
0 \leqslant D_{i} \leqslant 2.0835 \times 10^{-4}, \quad i=0, \ldots, 4 .
$$

We assume no exercise here, i.e., $u_{e x}(t)=0, t \in[0,540]$.

As shown in Figure 4, the resulting blood glucose level tracks the desired blood glucose profile reasonably well. Note from Table XI that the optimal solution involves a combination of 2 insulin types. The optimal objective function value for this case is 227.6.

\subsection{Case 3: Composite model with exercise}

All details for this case are the same as those for Case 2, except that we now also allow exercise. Unfortunately, the model appears to have multiple locally optimal solutions and we found it difficult to generate a better solution than that for Case 2 with a variety of random initial guesses of the insulin dosages and exercise levels. However, using the optimal insulin dosage levels and injection times 


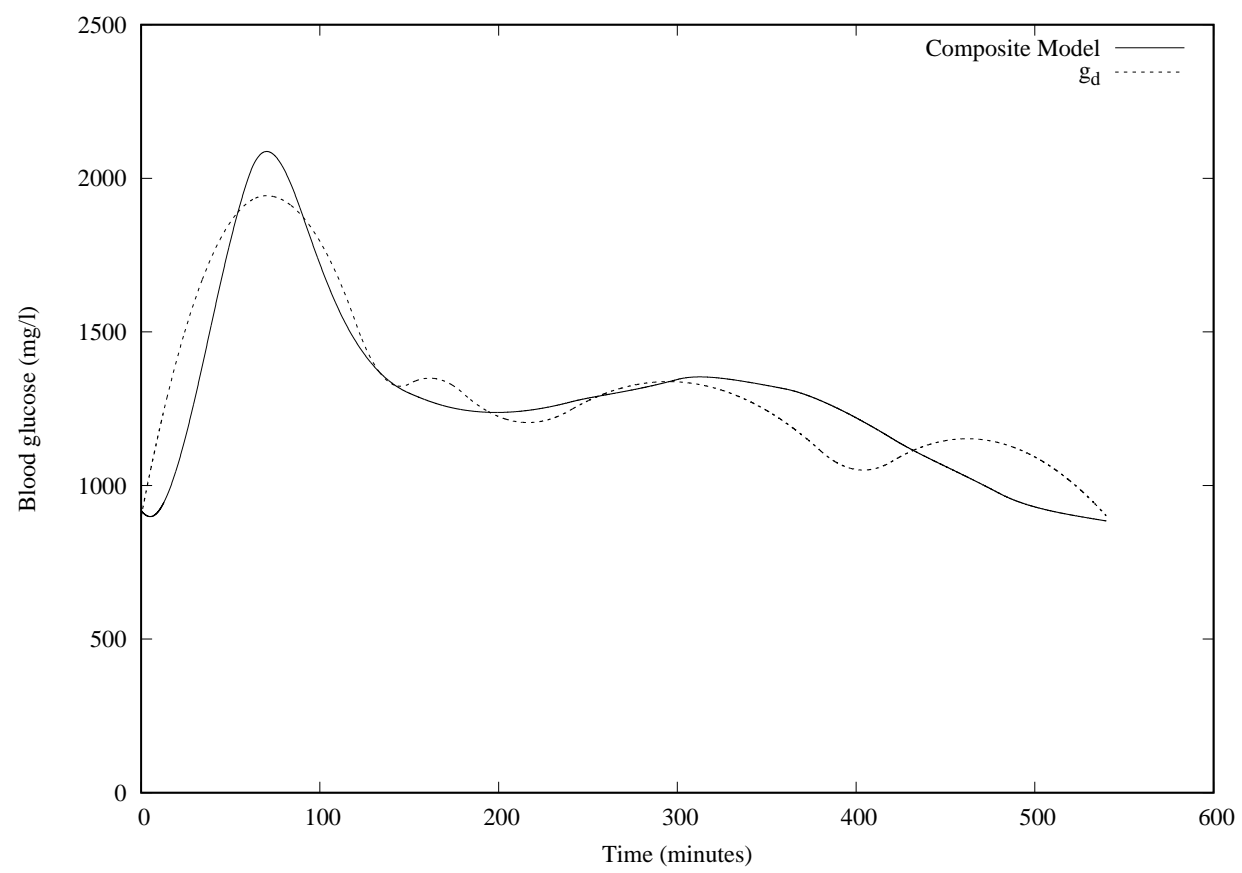

Figure 4. Blood glucose levels resulting from optimization of the model without exercise for Case 2.

\begin{tabular}{cccc}
\hline Dosages $D_{i}$ & Optimal values & Times $t_{i}$ & Optimal values \\
\hline$D_{0}$ & $6.68787 \times 10^{-05} \mathrm{M} \equiv 9.6 U$ & $t_{0}$ & 0 \\
\hline$D_{1}$ & $6.23415 \times 10^{-05} \mathrm{M} \equiv 9.0 U$ & $t_{1}$ & 134.984 \\
\hline$D_{2}$ & $1.17169 \times 10^{-05} \mathrm{M} \equiv 1.7 U$ & $t_{2}$ & 199.163 \\
\hline$D_{3}$ & $0 M \equiv 0 U$ & $t_{3}$ & 325.285 \\
\hline$D_{4}$ & $1.02286 \times 10^{-05} \mathrm{M} \equiv 1.5 U$ & $t_{4}$ & 450.921 \\
\hline
\end{tabular}

Table XII. Optimal values of decision variables $D_{i}$ and $t_{i}$ in Case 3.

from Case 2, along with a zero initial guess for the exercise level, did readily yield an improved solution as shown in Figure 5. Figure 6 shows the corresponding optimal exercise level and Table XII shows the optimal values of decision variables $D_{i}$ and $t_{i}, i=0, \ldots, 4$. Note that exercise is used for most of the time horizon in conjunction with lower doses of insulin injections. The optimal objective function value for this case is 122.4 .

\subsection{Case 4: Composite model for type 2 diabetes}

In order to test the composite model for a type 2 diabetic, we halve the original insulin feedback rate $R_{m}$. Here exercise is once again considered and the same types of insulin combinations as used in Cases 2 and 3 are assumed.

Clearly, Figure 7 shows that the resulting blood glucose level tracks the desired blood glucose profile very closely. Figure 8 shows the corresponding optimal exercise level. Also, the optimal dosages and times for the insulin injections are listed in Table XIII. Note that a much lower dosage 


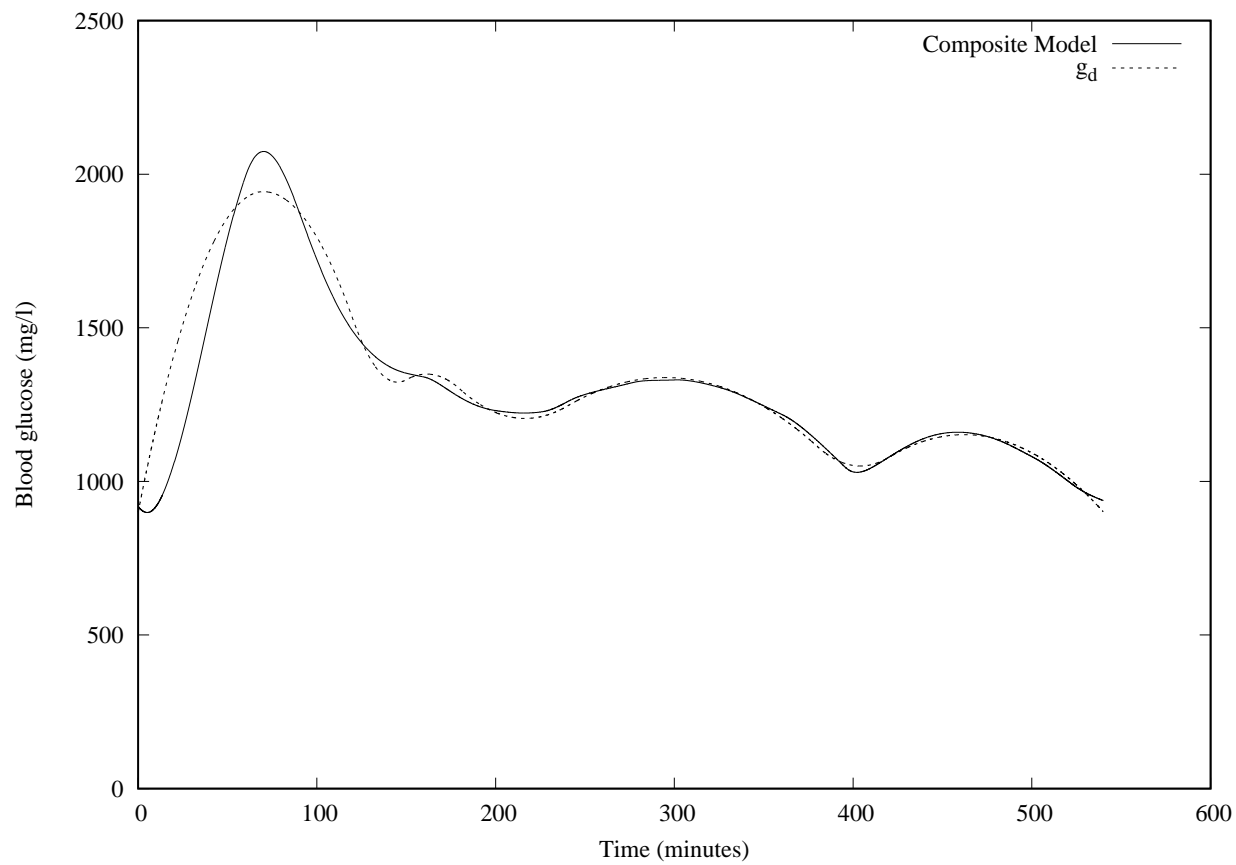

Figure 5. Blood glucose trajectories for Case 3.

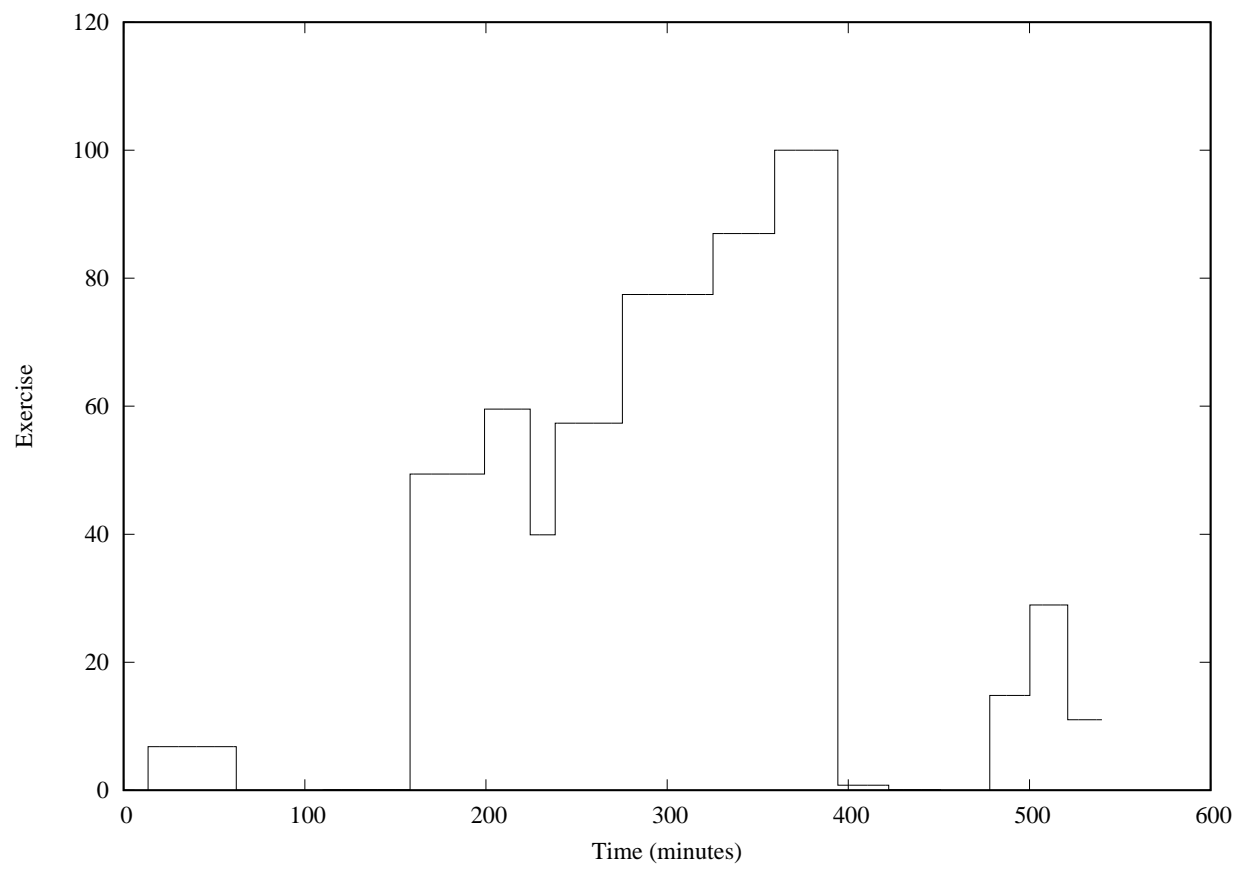

Figure 6. Optimal exercise level for Case 3. 


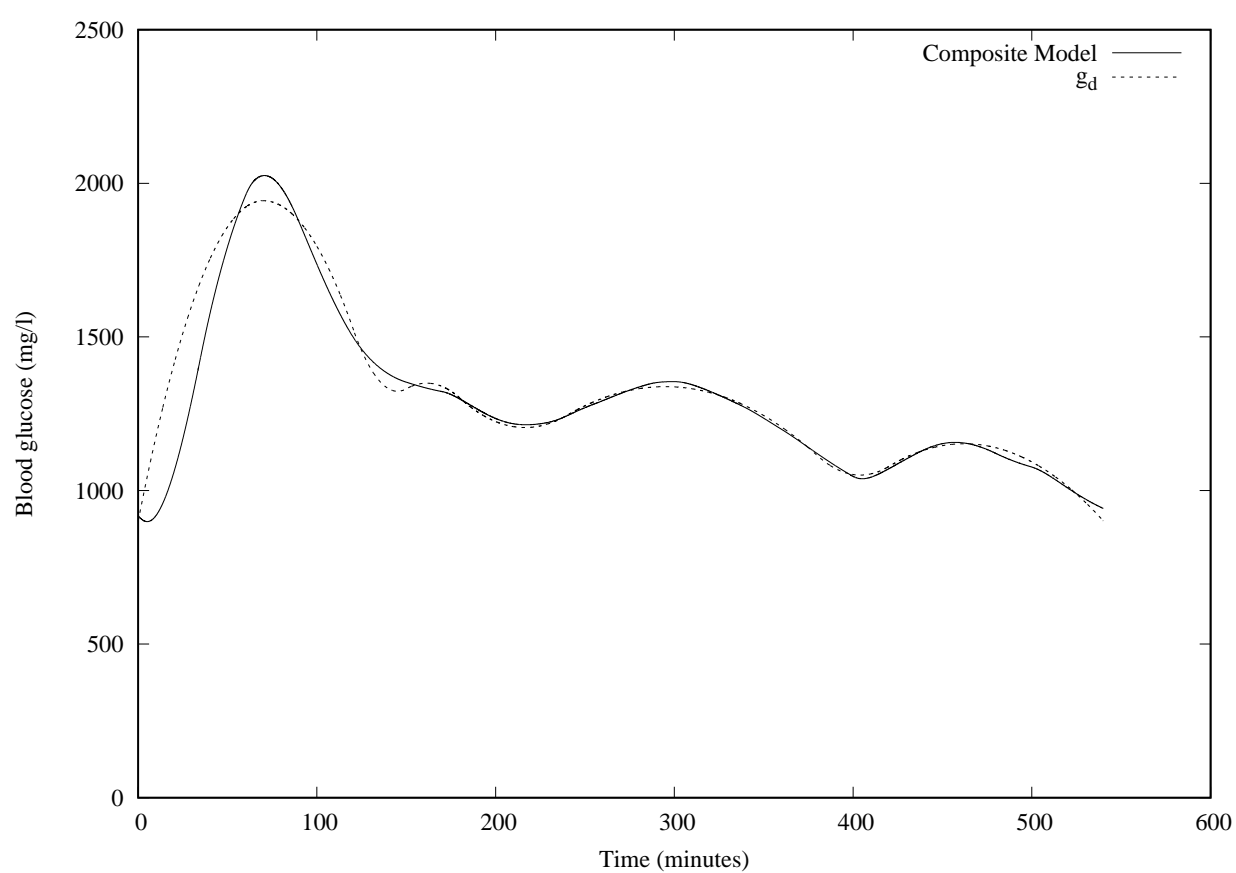

Figure 7. Blood glucose trajectories for Case 4.

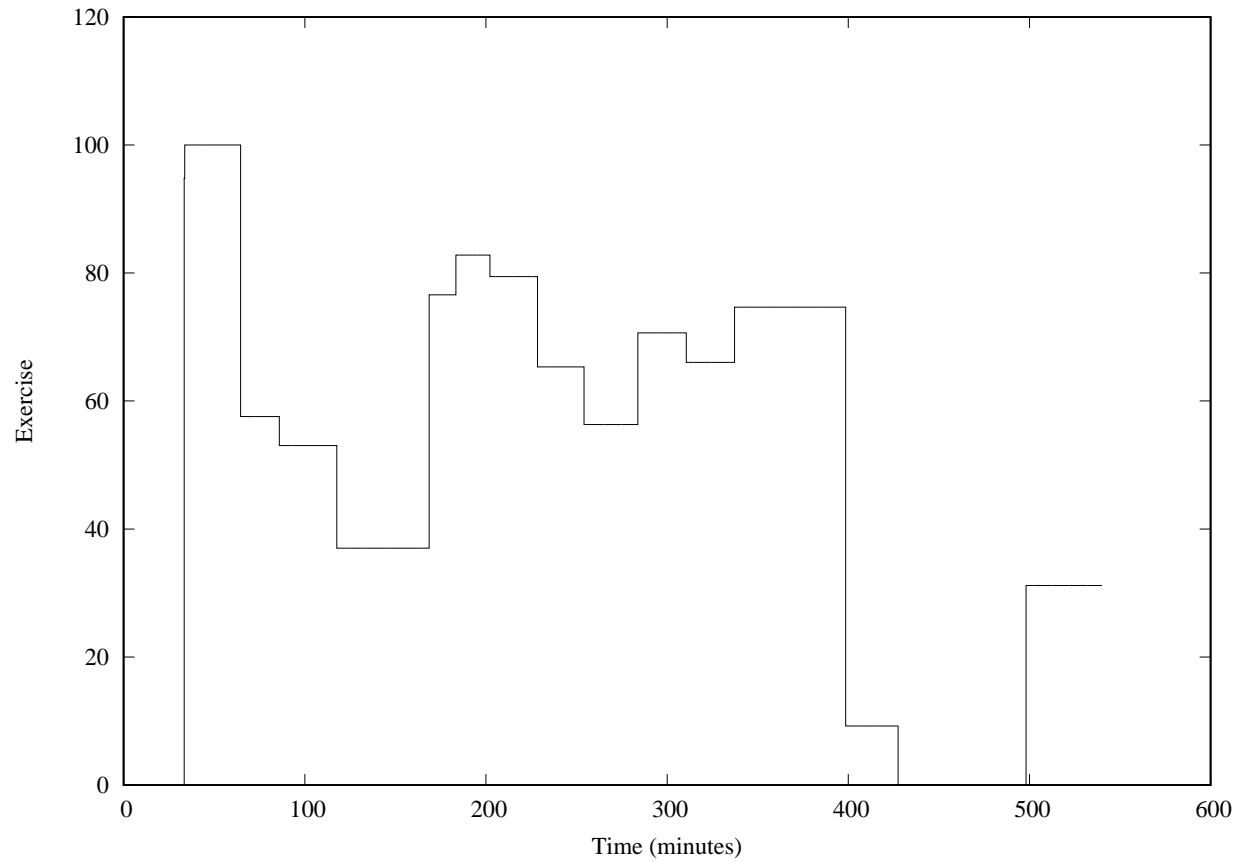

Figure 8. Optimal exercise level for Case 4. 


\begin{tabular}{cccc}
\hline Dosages $D_{i}$ & Optimal values & Times $t_{i}$ & Optimal values \\
\hline$D_{0}$ & $3.82462 \times 10^{-7} M \equiv 0.06 U$ & $t_{0}$ & 0 \\
\hline$D_{1}$ & $3.71972 \times 10^{-5} M \equiv 5.4 U$ & $t_{1}$ & 33.769 \\
\hline$D_{2}$ & 0 & $t_{2}$ & 168.681 \\
\hline$D_{3}$ & 0 & $t_{3}$ & 254.137 \\
\hline$D_{4}$ & 0 & $t_{4}$ & 398.603 \\
\hline
\end{tabular}

Table XIII. Optimal values of decision variables $D_{i}$ and $t_{i}$ in Case 4 .

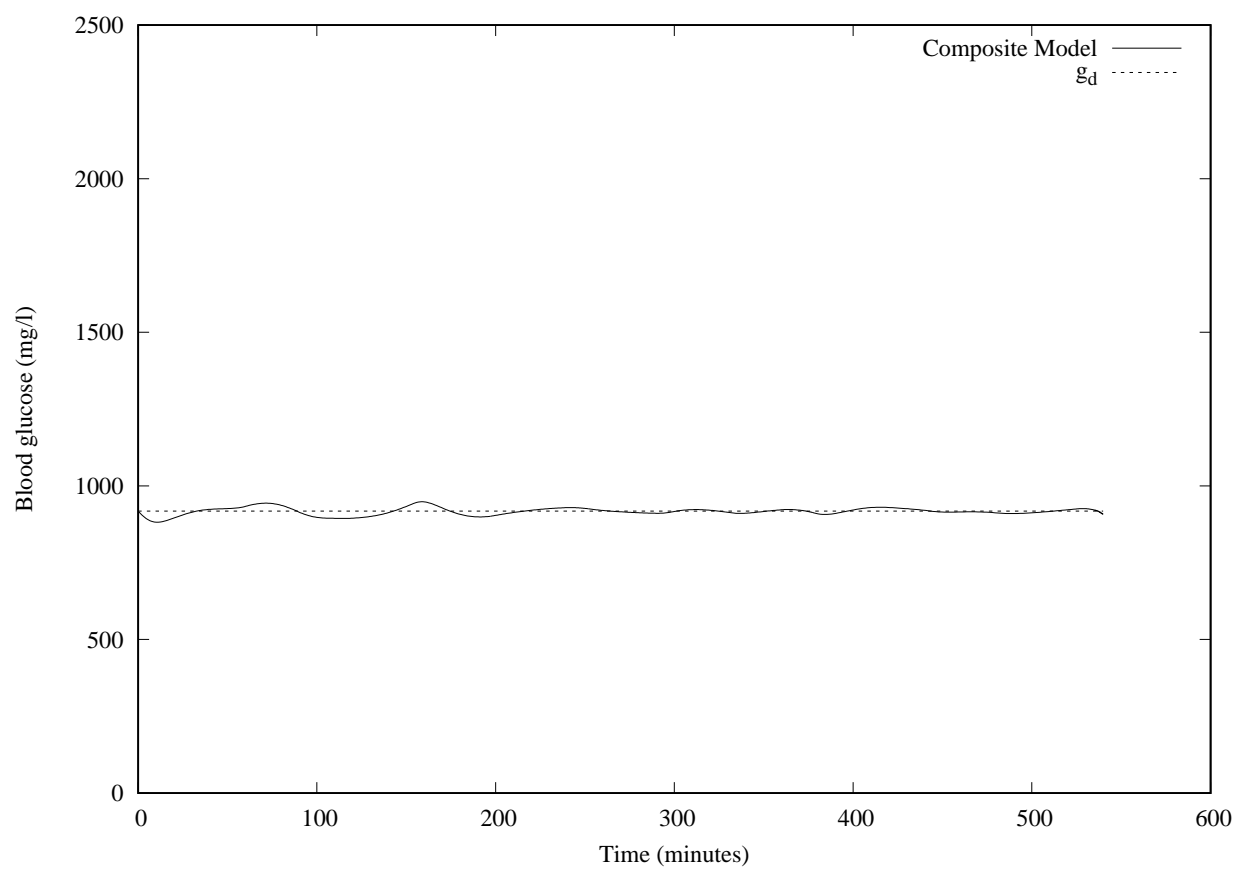

Figure 9. Blood glucose trajectories for Case 5.

of insulin along with an earlier use of exercise is sufficient to yield good blood glucose control in this case. The objective function value in this case is 107.8.

\subsection{Case 5: Aiming for basal blood glucose level}

In this case, we once again consider a type 1 diabetic $\left(R_{m}=0\right)$ and we adopt the objective function

$$
\tilde{J}=\int_{0}^{N} v(s)\left(\tilde{x}_{8}(s)-918\right)^{2} d s
$$

which simply penalizes any blood glucose deviation from the initial level. The problem is to minimize (72) subject to the same dynamics and constraints as in previous cases. All details for this case are the same as those of Case 2, except for the objective function and the assumed order of the insulin injections. We again assume that insulin injections have an alternating pattern, but this time we start with a short acting insulin.

As can be seen from Figure 9, the optimal blood glucose level resulting in this case remains very close to the initial blood glucose level $(918 \mathrm{mg} / \mathrm{l}=5.1 \mathrm{mmol} / \mathrm{l})$ over the entire time horizon. 


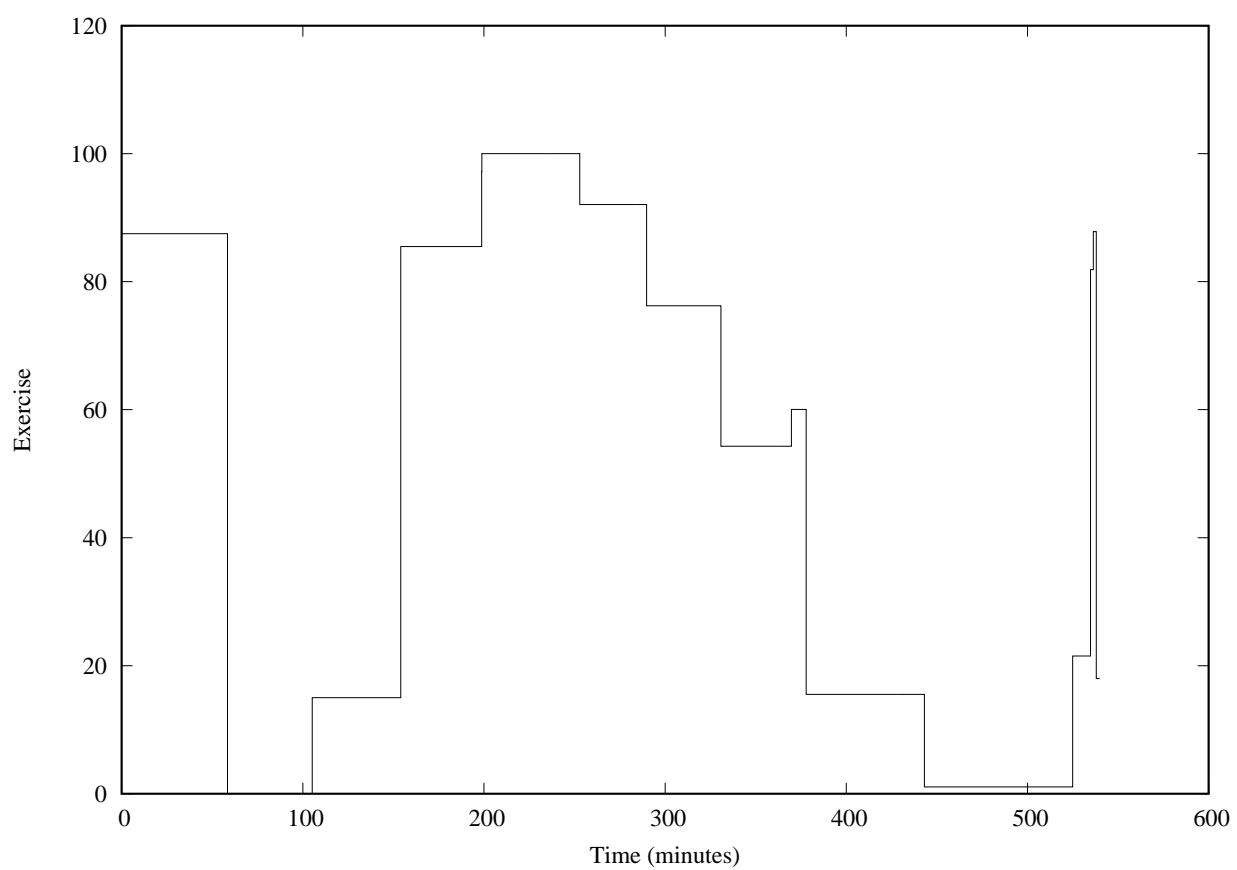

Figure 10. Optimal exercise level for Case 5.

\begin{tabular}{cccc}
\hline Dosages $D_{i}$ & Optimal values & Times $t_{i}$ & Optimal values \\
\hline$D_{0}$ & $1.28420 \times 10^{-04} M \equiv 18.5 U$ & $t_{0}$ & 0 \\
\hline$D_{1}$ & $7.28584 \times 10^{-06} M \equiv 1.05 U$ & $t_{1}$ & 198.672 \\
\hline$D_{2}$ & $4.40814 \times 10^{-05} M \equiv 6.35 U$ & $t_{2}$ & 198.736 \\
\hline$D_{3}$ & $2.29984 \times 10^{-06} M \equiv 0.33 U$ & $t_{3}$ & 330.677 \\
\hline$D_{4}$ & $3.77622 \times 10^{-05} M \equiv 5.44 U$ & $t_{4}$ & 524.814 \\
\hline
\end{tabular}

Table XIV. Optimal values of decision variables $D_{i}$ and $t_{i}$ in Case 5.

Figure 10 shows the optimal exercise level and Table XIV shows the optimal values of the decision variables $D_{i}$ and $t_{i}, i=0, \ldots, 4$. Due to the need to clear a lot of glucose early on, multiple insulin injections early in the time horizon combined with an early high level of exercise are required in this case. On the other hand, a very low objective function value of 2.53 is obtained.

\subsection{Robustness}

Open loop optimal controls may lack robustness with respect to noise and modelling errors, so we carry out a brief perturbation analysis for the optimal solution of Case 3 presented earlier. One possible source of uncertainty in the model is the exogenous glucose input rate corresponding to a meal. We used the data from Table I to generate the function $G$ shown in Figure 1 and employed in the model. We then perturbed each of the nonzero values in Table I sequentially by adding and subtracting $5 \%$ of its original value. For each of these 22 perturbations, we then applied the optimal control generated for Case 3 and observed the resulting blood glucose trajectory. These are collectively graphed in Figure 11. As can be clearly seen, the perturbed trajectories do not differ 


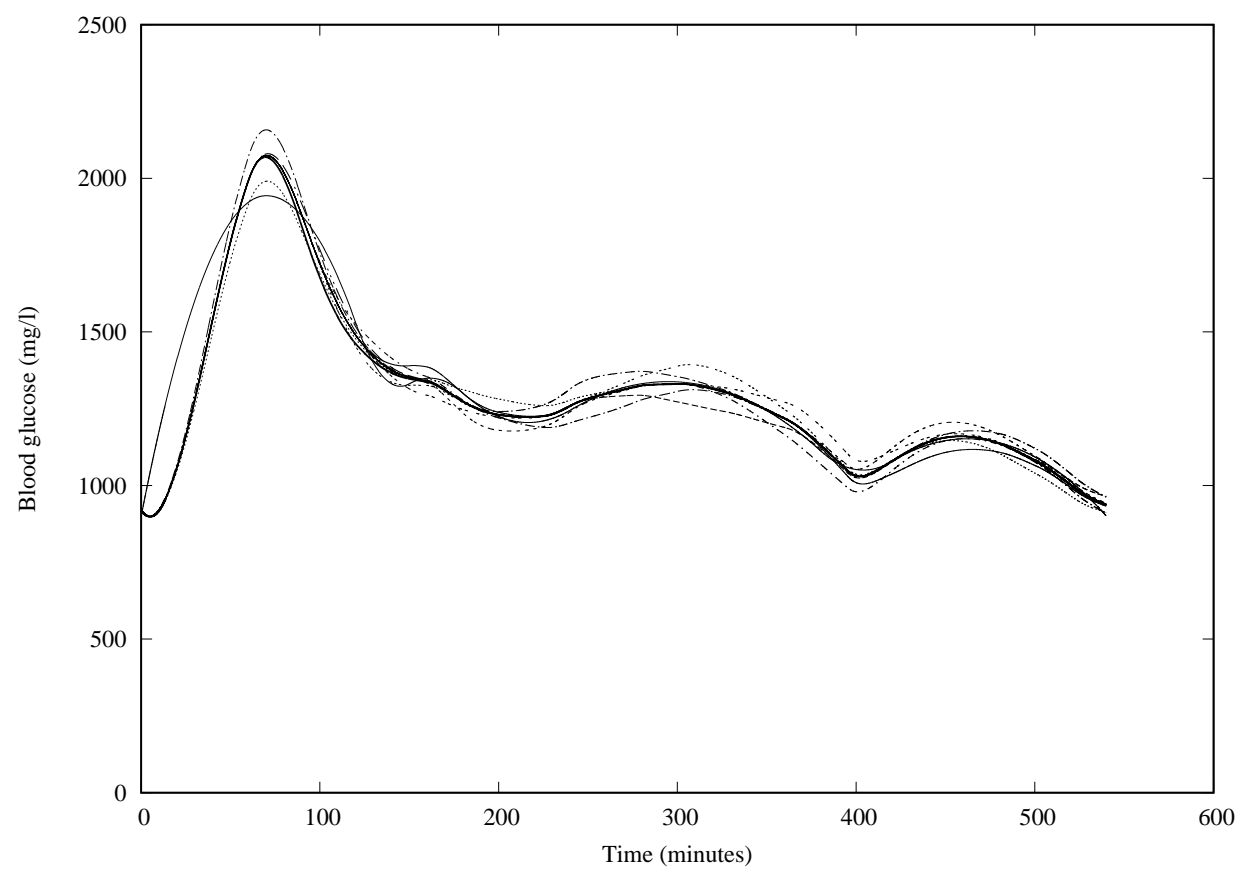

Figure 11. Perturbed trajectories for the optimal solution in Case 3.

greatly from the nominal trajectory and still yield good blood glucose control. Indeed, the resulting objective function values range from $99.1 \%$ to $111 \%$ of the nominal objective value.

\section{CONCLUSIONS}

We have extended an existing model of the blood glucose regulatory system by incorporating the effects of exercise and subcutaneous insulin injections. We have shown that optimal exercise regimes and insulin injection dosages and times can be readily determined using numerical optimal control methods, where the latter required the use of a time scaling transformation.

There is significant scope for future work. For example, just as we allowed for multiple insulin injection times, it should be possible to incorporate multiple meals and also optimize the timing of these meals. The optimal results in Section 5 involve extended periods of very high levels of exercise, which is unlikely to be achievable in practice. Although one could easily reduce the upper bound on $u_{e x}(t)$ in (44), a more reasonable approach would be to limit the total exercise effort measured by $\int_{0}^{T} u_{e x}(t) d t$. This type of integral constraint can easily be included in the optimal control formulation and computation [12]. While the blood glucose levels in our results did not get anywhere close to practical minimum or maximum limits, more general formulations of the problem should also impose upper and lower bounds on the blood glucose level over the entire time horizon. Again, these types of all time state constraints can be readily incorporated into the optimal control formulation [12]. Another interesting challenge would be the incorporation of free fatty acids (FFAs) into the model [23], since these also represent an important energy source for the body. One should also investigate the possibility of incorporating other types of treatments typical for type 2 diabetics. Finally, note that any of the extended models will contain many model constants which need to be chosen appropriately. Since individual subjects can respond quite differently to a range of factors impacting on blood glucose levels, it is advisable to choose these model constants for each 
individual so that the resulting model follows the available experimental data for that individual as closely as possible. The formulation and numerical solution of an appropriate optimal parameter selection problem as described in [9] and [14] is a practical way to accomplish this task.

\section{REFERENCES}

1. Ackerman E, Gatewood L, Rosevear J, Molnar GD. Model studies of blood-glucose regulation. The Bulletin of Mathematical Biophysics 1965; 27(1):21-37.

2. Fridlyand L, Philipson L. Glucose sensing in the pancreatic beta cell: A computational systems analysis. Theoretical Biology and Medical Modelling 2010; 7.

3. Man C, Rizza R, Cobelli C. Meal simulation model of the glucose-insulin system. Biomedical Engineering, IEEE Transactions on 2007; 54(10):1740-1749.

4. Bergman R, Phillips L, Cobelli C. Physiologic evaluation of factors controlling glucose tolerance in man: measurement of insulin sensitivity and beta-cell glucose sensitivity from the response to intravenous glucose. Journal of Clinical Investigation 1981; 68(6):1456-1467.

5. Cobelli C, Federspil G, Pacini G, Salvan A, Scandellari C. An integrated mathematical model of the dynamics of blood glucose and its hormonal control. Mathematical Biosciences 1982; 58(1):27-60.

6. Fisher M, Teo K. Optimal insulin infusion resulting from a mathematical model of blood glucose dynamics. Biomedical Engineering, IEEE Transactions on 1989; 36(4):479-486.

7. Fisher M. A semiclosed-loop algorithm for the control of blood glucose levels in diabetics. Biomedical Engineering, IEEE Transactions on 1991; 38(1):57-61.

8. Liu W, Tang F. Modeling a simplified regulatory system of blood glucose at molecular levels. Journal of Theoretical Biology 2008; 252(4):608-620.

9. Al Helal Z, Rehbock V, Loxton R. Modelling and optimal control of blood glucose levels in the human body. Journal of Industrial and Management Optimization 2015; 11(4):1149-1164.

10. Lin Q, Loxton R, Teo K. The control parameterization method for nonlinear optimal control: a survey. Journal of Industrial and Management Optimization 2014; 10(1):275-309.

11. Loxton R, Teo K, Rehbock V. Optimal control problems with multiple characteristic time points in the objective and constraints. Automatica 2008; 44(11):2923-2929.

12. Jennings L, Fisher M, Teo K, Goh C. MISER3 Optimal Control Software: Theory and User Manual. The University of Western Australia, Perth July 2004.

13. Korach-André M, Roth H, Barnoud D, Péan M, Péronnet F, Leverve X. Glucose appearance in the peripheral circulation and liver glucose output in men after a large 13C starch meal. The American Journal of Clinical Nutrition 2004; 80(4):881-886.

14. Lin Q, Loxton R, Xu C, Teo K. Parameter estimation for nonlinear time-delay systems with noisy output measurements. Automatica 2015; 60:48-56.

15. Kobayashi T, Sawano S, Itoh T, Kosaka K, Hirayama H, Kasuya Y. The pharmacokinetics of insulin after continuous subcutaneous infusion or bolus subcutaneous injection in diabetic patients. Diabetes 1983; 32(4):331-336.

16. Trajanoski Z, Wach P, Kotanko P, Ott A, Skraba F. Pharmacokinetic model for the absorption of subcutaneously injected soluble insulin and monomeric insulin analogues. Biomedizinische Technik. Biomedical Engineering 1993; 38(9):224-231.

17. Nucci G, Cobelli C. Models of subcutaneous insulin kinetics. A critical review. Computer Methods and Programs in Biomedicine 2000; 62(3):249-257.

18. Berger M, Rodbard D. Computer simulation of plasma insulin and glucose dynamics after subcutaneous insulin injection. Diabetes Care 1989; 12(10):725-736.

19. Derouich M, Boutayeb A. The effect of physical exercise on the dynamics of glucose and insulin. Journal of Biomechanics 2002; 35(7):911-917.

20. Breton M. Physical activity - the major unaccounted impediment to closed loop control. Journal of Diabetes Science and Technology 2008; 2(1):169-174.

21. Hernández-Ordoñez M, Campos-Delgado D. An extension to the compartmental model of type 1 diabetic patients to reproduce exercise periods with glycogen depletion and replenishment. Journal of Biomechanics 2008; 41(4):744752.

22. Sorensen J. A physiologic model of glucose metabolism in man and its use to design and assess improved insulin therapies for diabetes. PhD Thesis, Chemical Engineering Department, Massachusetts Institute of Technology, Cambridge 1985.

23. Roy A, Parker RS. A phenomenological model of plasma FFA, glucose, and insulin concentrations during rest and exercise. American Control Conference, 2010; 5161-5166. 